\title{
Successful Aging and Longevity in Older Old Women: The Role of Depression and Cognition
}

\author{
Daniel Paulson, ${ }^{1}$ Mary Elizabeth Bowen, ${ }^{2}$ and Peter A. Lichtenberg1 \\ ${ }^{1}$ Department of Psychology and Institute of Gerontology, Wayne State University, Detroit, MI 48202-3801, USA \\ ${ }^{2}$ Veterans Health Administration, HSR\&D/RR\&D Center of Excellence, Tampa, FL 33637-1022, USA \\ Correspondence should be addressed to Daniel Paulson, paulson@wayne.edu
}

Received 15 March 2011; Revised 2 May 2011; Accepted 17 May 2011

Academic Editor: B. A. Hagberg

Copyright ( 2011 Daniel Paulson et al. This is an open access article distributed under the Creative Commons Attribution License, which permits unrestricted use, distribution, and reproduction in any medium, provided the original work is properly cited.

\begin{abstract}
Based in successful aging theory and terminal cognitive drop research, this paper investigates cerebrovascular burden (CVB), depressive symptoms, and cognitive decline as threats to longevity. A subsample of stroke-free women over the age of 80 was identified in the Health and Retirement Survey (years 2000-2008). Mortality at 2, 6, and 8 year intervals was predicted using CVB (diabetes, heart disease, hypertension), depressive symptoms (Center for Epidemiological Studies Depression Scale), and cognitive decline (decline of 1 standard deviation or more on the 35-point Telephone Interview for Cognitive Status over 2 years). At most waves (2002, 2004, and 2006) mortality was predicted by CVB, depressive symptoms, and cognitive drop measured 2 years prior. CVB and depressive symptoms at the 2000 wave predicted mortality at 6 and 8 years. Older women with the greatest longevity had low CVB, robust cognitive functioning, and few depression symptoms, supporting successful aging theory and terminal cognitive drop.
\end{abstract}

\section{Introduction}

Rowe and Kahn [1] proposed criteria for successful aging comprised of avoidance of disease, maintenance of high cognitive and physical function, and sustained engagement in social and productive activities. This model grew from highly prolific MacArthur Foundation Study of Successful Aging, a $\$ 10$ million, 10-year research effort led by Rowe and Kahn. The objectives of this study, and the theoretical framework that grew from it, are to better understand risk factors for decline and to inform prevention efforts. For instance, work drawn from this initiative concluded that pulmonary health relates to both gross motor and cognitive functioning in late life, suggesting this as an area for primary intervention in preserving late-life independence [2]. Drawing on the MacArthur Study data, Others have investigated modifiable risk factors for dementia, concluding that late-life depression may be a precursor of cognitive decline [3]. Still other work based in this study found that older adults who frequently felt useful to others had lower rates of disability and mortality than less-engaged elders, emphasizing the importance of social engagement and productive activities [4]. While support for this theory of successful aging has been mixed $[5,6]$, it provides a useful framework for examining longevity. This paper will examine whether the Rowe and Kahn successful aging variables are each independently related to longevity. In the current paper we chose to examine this theory in older old women over 80 years. Women constitute a majority of all older adults over 80 but more importantly, they are more likely than men to experience disability and to live more years with disability than men [7]. Geriatric syndromes such as cardiovascular disease, cognitive decline, and depression compromise functional independence more with increasing age [8]. Age 80 represents a point when medical treatment planning for elders should be reevaluated given the escalating risks associated with these comorbidities.

Behavioral factors as defined in this paper include psychological aspects of functioning that can be measured through self-report or performance-based assessment, such as mood and cognitive functioning (i.e., depression and cognition). Important behavioral factors such as cognition and depression represent major risk factors for disability, often preceding disability [9] and possibly reducing longevity. Serious disability, especially mobility loss, has been linked to reduced survival [10]. Identifying behavioral factors that 
hasten disability onset may then lead to improved models of integrated care. Behavioral factors such as depression and cognitive decline may best be understood when integrated with chronic disease, especially those that enhance vascular risk.

Though Rowe and Kahn interpreted "avoidance of disease" broadly, vascular disease is particularly significant to healthy aging as these chronic conditions (e.g., hypertension, atrial fibrillation, and diabetes) gradually compromise adaptive resources. Neural network functioning is broadly compromised by the effect of vascular disease on cerebral tissue, termed cerebrovascular burden (CVB). CVB is also associated with higher stroke risk, cardiac disease, and sensorimotor impairment. Moreover, high CVB hastens the manifestation of clinically significant cognitive impairment, regardless of the specific etiology of cognitive decline (e.g., normative aging, Alzheimer's disease, Parkinson's disease, and vascular dementia) [11]. Elders with high CVB tend to have less prefrontal white matter volume, more white matter hyperintensities, and comparably impaired executive functioning [12]. Considerable evidence also exists that CVB also contributes to the development of late-life depression symptoms $[13,14]$. Thus, in addition to being a broad measure of physical health in the Rowe and Kahn model, inclusion of CVB will distinguish variance in mortality risk directly related to this variable, thereby providing a more stringent test of how depression and cognition independently relate to longevity.

Successful aging theory identifies sustained engagement in social and productive activities as central to healthy aging. Clinical depression throughout the lifespan is characterized by reduced enjoyment in activities and decreased social engagement. As such, depression symptoms in late life represent a significant barrier to successful aging based on this interpretation. Generally speaking, more depression symptoms translate to poorer health outcomes. For instance, people experiencing depression are at greater risk of a first heart attack [15], stroke [16, 17], cancer [18], worse health outcomes after controlling for cardiovascular risks [15], and higher mortality [19]. Depression in later life was found to be a significant risk factor for death. Mehta et al. [20] reported that, in a large sample of community-dwelling elders, mortality was significantly predicted by both number of depressive symptoms and performance on a measure of cognitive functioning. Similarly, it was reported that in a sample of older patients with debilitating or chronic medical diagnoses, after controlling for age, comorbidity and illness severity, functional impairment and cognitive functioning, depressed respondents were $34 \%$ more likely to die over three years [21]. In a large $(N=3065)$ Dutch sample of individuals between the ages of 55 and 80 , depression was identified as a significant risk factor for death over four years. However, the strength of this effect was partially eroded by the addition of other variables such as chronic disease, smoking, and physical inactivity [22].

The third domain of successful aging theory identifies preservation of cognitive and physical functioning as critical to successful aging. Cognitive impairment limits quality of life by reducing the capacity for meaningful work and social interaction [23], and rapid loss of cognitive faculties often suggests medical decline with a heightened mortality risk $[22,24]$. Terminal cognitive drop is identified as an accelerated loss of cognitive functioning preceding death [24], by contrast to terminal decline which is a linear decline function preceding death [25]. A review from 2002 concluded that, largely because testing terminal drop theory requires a repeated-measures design, limited data existed supporting this theory [26]. Since this review, several longitudinal studies have been published, including a recent study concluding that elders with sharp declines on the Minimental State Exam [27], a brief cognitive screening measure, experienced more activity of daily living (ADL) disability and higher mortality rates than elders with more stable scores [28]. While estimates of the temporal relationship between terminal drop and death vary, recent research identified evidence of terminal drop at a mean of 42 months before death [29] in a large sample of dementia-free elders. These estimates are roughly similar to the original estimates of about 5 years reported by K. F. Riegel and R. M. Riegel [24]. Similarly, past work drawing on the Health and Retirement Survey (HRS) data identified a relationship between cognitive impairment and mortality over a 2-year interval [30]; however, this study evaluated cognitive decline crosssectionally and did not include other markers of decline such as CVB or depression. Of note, Langa et al. reported evidence of compression of cognitive morbidity; elders with moderate or severe cognitive impairment in 2002 had greater risk of death over 2 years than those with similar levels of impairment in 1993.

Comorbid cognitive impairment and depressive symptoms suggest particularly high risk of death $[19,20]$; however, these studies evaluated cognitive functioning crosssectionally. While impairment on cognition measures suggests decline from demographically representative norms, the concept of terminal cognitive drop specifies rapid loss of cognitive functioning over a brief period of time. Relatively few studies relating to cognition and longevity evaluate how decline over brief periods relates to longevity, and even fewer investigate whether depression and cognitive decline are independent predictors of mortality. Because cognition and mood, domains in which impairment may be most evident to family and medical practitioners alike, tend to be interrelated in late life $[31,32]$, it is important to distinguish the individual relationships of these variables with longevity.

\section{Objectives of the Present Study}

This investigation seeks to examine whether all three domains of Rowe and Kahn's successful aging paradigm independently predict longevity in a large sample of stroke-free women over the age of 80 . In particular, this study emphasizes two behavioral domains that are of particular relevance in clinical settings-depression and cognitive decline. This theoretical orientation, based on a broad reading of the literature, posits that elders with few depression symptoms and preserved cognitive functioning will experience greater longevity. In addition to CVB, other health variables such as self-rated health and Body Mass Index (BMI) will be 
included in order to control for general health. The model proposed predicts that both depression and cognitive decline will predict mortality in this sample. Hypothesis 1 looks at survival across the entire study period of 8 years, whereas Hypothesis 2 examines proximate predictors of death across 2 -year intervals.

Hypothesis 1. Physical health, depressive symptoms, and cognitive decline at baseline (2000 wave) will all be independent predictors of longevity conceptualized as survival across the entire 8-year study period.

Hypothesis 2. Incidence of death at each wave (2002, 2004, 2006, and 2008) will be predicted by CVB, cognitive decline, and depressive symptoms at the previous wave.

\section{Methodology}

3.1. Sample. The Health and Retirement Survey (HRS) is a prospective cohort study conducted by the University of Michigan with support from the National Institute on Aging. The first wave of the HRS occurred in 1992 with a 51-to61 year-old cohort and was merged with the older (70 years and older) Asset and Health Dynamics of the Oldest Old Study (AHEAD) cohort in 1998. Two additional cohorts were added in 1998 to fill in the gaps between these two groups. Briefly, the HRS is a multistage probability cohort sample of US households. Further details on the HRS design and methods have been previously published [33].

The present study utilized the Health and Retirement Survey (HRS) that was prepared by the RAND Center for the Study of Aging (RAND HRS). The selected portion of this publically available, longitudinal dataset includes five waves at two-year intervals from 2000 through 2008 (waves 5 through 9). Inclusion criteria included age over 80 years at the first wave and female sex. This study made use of the 1998 TICS score to identify incidence of cognitive decline from 1998 to 2000. Because stroke is associated with highly variable cognitive performance, participants with history of stroke prior to 1998 were excluded. Respondents who were unable to complete survey materials at the 1998 data collection were also excluded as missing data would have precluded calculation of 1998-2000 cognitive change scores. This data set is demographically representative of the female US population over age 80 .

\subsection{Measures}

3.2.1. Medical Data. Medical data (hypertension, diabetes, history of heart disease, height, and weight) and lifetime history of smoking were collected by self-report. CVB was identified as the number of cerebrovascular risk factors (hypertension, diabetes, and history of heart disease) reported creating a score ranging from 0 to 3 .

3.2.2. Depressive Symptoms. A shortened, 8-item form of the original Center for Epidemiological Studies Depression Scale (CESD) was used to evaluate depression [34]. Six of the eight items are negatively worded, and two are positively worded. Participants are asked to respond "yes" or "no" to each item ("was depressed," "everything was an effort," "sleep was restless," "was happy," "felt lonely," "enjoyed life," "felt sad," "could not get going") that occurred within the preceding week. Scores ranged from 0 to 8 with higher scores indicating greater depressive symptoms. Using HRS data, the reliability of the 8 -item CESD measure was adequate, with Cronbach's alpha ranging from .81 to .83 between waves [35]. High validity and symptom dimensions similar to those in the longer 20-item CESD have been demonstrated using these 8 items $[35,36]$. The CESD is broadly used in the epidemiological study of late-life depression [37]. While the CESD literature describes a clinical cutoff that can be used to distinguish respondents with probable depression[35], this measure was used as a semicontinuous variable representing the full range depressive symptoms in this population sample.

3.2.3. Cognitive Functioning. The HRS data includes a brief standardized 35-point measure of cognitive functioning that was developed for remote screening of cognitive disorders based on the Telephone Interview for Cognitive Status [38]. It includes indices of orientation, concentration, short-term memory, mathematical skills, praxis and language and has a maximum score of 35 points (observed range: $0-35$ ) with higher scores reflecting better functioning. This instrument has a Cronbach's alpha of.69 and past work has identified factors reflecting mental status and memory [39]. The TICS has demonstrated high test-retest reliability and is generally sensitive to cognitive impairment [38, 40-42]. For all of the following models cognitive decline was identified as a decrease in TICS score from one wave to the next wave of more than one standard deviation (6.1 points) based on baseline data.

3.2.4. Self-Rated Health. Self-rated health change was measured with a single question assessing the respondents' perception of change in health since the last data collection 2 years prior. Change in self-rated health was assessed with the question, "Compared to your health when we talked with you in (last wave) would you say that your health is better now, about the same, or worse?" Response options included "much better," "somewhat better," "same," "somewhat worse," "much worse," comprising a 5-point scale. This variable was included as a semicontinuous measure.

\section{Statistical Methodology}

Binary logistic regression was performed to model the likelihood of death between baseline (2000) and either 2006 or 2008 as a function of demographics, cognitive function, CVB, and depression. While the study spans the years 20002008, the logistic regression using 2006 as an end point was included as closer to half of the sample had died at this wave, thereby optimizing the statistical power of the model. Variables were entered blockwise with age, years of education, and body mass index (BMI) entered in the first block. The second block included CVB as reported in the 2000 wave. 
TABLE 1: Sample description at the 2000 wave (baseline).

\begin{tabular}{lcc}
\hline Variable & Mean & SD \\
\hline Age & 85.63 & 3.82 \\
Education & 11.03 & 3.42 \\
\hline CVB & 1.03 & 0.82 \\
CESD & 2.07 & 1.98 \\
TICS & 18.13 & 5.86 \\
BMI & 24.40 & 4.86 \\
Self-rated health change & 3.36 & 0.83 \\
\hline Ethnic distribution & Percentage & \\
White & $79.7 \%$ & \\
Black & $14.2 \%$ & \\
Hispanic & $5.2 \%$ & \\
Other & $1.1 \%$ & \\
\% high CVB & $42.2 \%$ & \\
\hline
\end{tabular}

Note. CVB: number of symptoms comprising cerebrovascular burden (heart disease, diabetes, hypertension scored 0-3). CESD: Center for Epidemiological Studies Depression Scale, TICS: Telephone Interview for Cognitive Status, BMI: Body Mass Index.

The third block included the self-rated health change variable at the 2000 wave. The fourth block included CESD score at the 2000 wave and a variable reflecting incidence of decline greater than 1 standard deviation ( $>6$ points) on the TICS between the 1998 and 2000 waves.

To better track the relationship between TICS score decline, mood, and longevity over the course of the study, four additional logistic regression models were computed. In the first, incidence of death in 2002 was predicted using $2000 \mathrm{CVB}$, the $2000 \mathrm{CESD}$ score, and the index reflecting a drop in TICS score between 1998 and 2000 exceeding 1 standard deviation. The second model predicted death in 2004 based on CVB in 2002, CESD score in 2002, and incidence of decline in TICS score of more than 1 standard deviation between 2000 and 2002. The third and fourth models predicted death in 2006 and 2008 using similar predictors, respectively.

\section{Results}

Of the 1368 respondents who met criteria at the 1998 wave, 1186 were living at the 2000 wave. Of these, 417 were living at the 2008 wave representing a $64.8 \%$ mortality rate over this 8 -year period. The sample at the 2000 wave is described in Table 1 . The mean age was 85.6 years $(\mathrm{SD}=3.8)$. On average, respondents had 11 years of formal education $(\mathrm{SD}=3.4)$. The mean BMI at baseline was $24.4(\mathrm{SD}=4.9)$. The average number of cerebrovascular risk factors reported was 1 ( $\mathrm{SD}=$ 0.8). The mean CESD score was $2.1(\mathrm{SD}=2)$ suggesting a low rate of depressive symptoms in this population. The mean score on the 35 TICS measure was 18.1 ( $\mathrm{SD}=5.9)$.

As can be seen in Table 2, CVB and depressive symptoms were significant predictors of mortality between 2000 and 2006. In addition, age, and BMI were also significant predictors. Cognitive decline and self-rated health showed a trend toward being significant predictors. A slightly different picture emerged with respect to 2008 outcomes. CVB was a significant predictor of mortality, and again BMI and age also predicted mortality. Depressive symptoms showed a trend toward significance, while self-rated health was not a significant predictor. Cognitive decline between 1998 and 2000 was not a significant predictor of mortality at the 2008 wave. Overall, the logistic regressions over 6 and 8 years provided partial support for applying Rowe and Kahn's successful aging model to longevity.

Four additional logistic regressions predicting death in 2002, 2004, 2006, and 2008 were completed to better understand the relationship between CVB, mood, cognitive change, and longevity over brief periods of time. CESD and TICS scores are unavailable for many participants, primarily for reasons of incapacity. As a result of listwise deletion caused by absent data on these predictor variables, these four logistic regression analyses included 69\% (2002), 61\% (2004), 54\% (2006), and 43\% (2008) of respondents who died, respectively. As described in Table 3, CVB significantly predicted mortality in the 2002, 2004, and 2008 waves. CESD score was a significant predictor of death in the 2002 and 2004 waves. Decline in TICS score over 2 years of more than 1 standard deviation significantly predicted incidence of death in 2004 and 2008 and showed a trend toward significance in $2002(P=.088)$.

\section{Conclusions}

The primary findings of the present research are that, among women over the age of $80, \mathrm{CVB}$, depressive symptoms, and rapid cognitive decline (terminal drop) predict incidence of mortality over brief periods (2 years). CVB significantly predicted 2-year mortality at 3 of 4 waves, and depressive symptoms and cognitive decline significantly predicted mortality at 2 of 4 waves. Over long periods ( 6 or 8 years) mortality was significantly predicted by age, BMI, and CVB. Additionally, depressive symptoms significantly predicted mortality over 6 years and showed a trend toward significance in predicting mortality over 8 years. Incidence of rapid cognitive decline was not a significant predictor of death over 6 or 8 years. As demonstrated in the analyses predicting death over 2year periods, rapid cognitive decline is a robust predictor of proximal death. Together, these findings suggest that, in addition to undermining quality of life and independence as described by Rowe and Kahn, sharp cognitive decline suggests high risk of imminent death. Cognitive drop, even in the older old is a significant indicator of declining health and potentially shortened life. While it is well documented that cognitive abilities decline in those over 80 [43], it is when declines are significantly above the norm when life expectancy is affected among the older old. By contrast, many of those who died toward the end of this study had robust cognitive functioning at this baseline interval. These results are consistent with past work describing the relationship between $\mathrm{CVB}$, mood, cognitive decline, and longevity [19, 20, 22, 24, 28, 29].

The present findings support and extend Rowe and Kahn's [1] successful aging theory by relating to low CVB, relatively few depressive symptoms, and preserved cognitive functioning, representing the three domains of successful 
TABLE 2: Results of logistic regression predicting incidence of mortality between 2000 and 2006.

\begin{tabular}{lcccccccc}
\hline & \multicolumn{3}{c}{ Predicting death between 2000 and 2006 } & \multicolumn{3}{c}{ Predicting death between 2000 and 2008} \\
Variable & $B$ & SE & Wald & $\operatorname{Exp}(B)$ 95\% CI & $B$ & SE & Wald & Exp $(B) 95 \%$ CI \\
\hline Age & 0.15 & 0.02 & $55.65^{\psi}$ & $1.12-1.21$ & 0.15 & 0.02 & $46.86^{\psi}$ & $1.11-1.22$ \\
Education & -0.03 & 0.02 & 1.61 & $.93-1.02$ & -0.03 & 0.02 & 1.54 & $.93-1.02$ \\
BMI & -0.07 & 0.02 & $17.27^{\psi}$ & $.91-.97$ & -0.07 & 0.02 & $18.76^{\psi}$ & $.90-.96$ \\
CVB & 0.46 & 0.09 & $26.74^{\psi}$ & $1.33-1.88$ & 0.56 & 0.09 & $36.19^{\psi}$ & $1.46-2.10$ \\
Self-rated health & 0.17 & 0.09 & $3.21^{\S}$ & $.98-1.42$ & 0.15 & 0.10 & 2.37 & $.96-1.41$ \\
2000 CESD score & 0.08 & 0.04 & $4.26^{*}$ & $1.00-1.16$ & 0.07 & 0.04 & $3.46^{\S}$ & $.99-1.16$ \\
1998-2000 TICS Drop & 0.35 & 0.21 & $2.81^{\S}$ & $.94-2.12$ & 0.05 & 0.22 & 0.06 & $.69-1.62$ \\
Constant & -12.00 & 1.82 & 43.44 & & -11.31 & 1.97 & 32.87
\end{tabular}

Note. BMI: Body Mass Index. CVB: cerebrovascular burden, self-rated health change: change in self-rated health from previous wave, CESD: Center for Epidemiological Studies Depression Scale, TICS: Telephone Interview for Cognitive Status. TICS Drop reflects incidence of decline on TICS score greater than 6 points between 1998 and 2000 .

$\S_{P}$ between .05 and $.10,{ }^{*} P<.05,{ }^{*} P<.001, \mathrm{df}=1$ for all comparisons.

TABLE 3: Results of logistic regression analyses predicting death at 2002, 2004, 2006, and 2008 waves based on depressive symptoms and incidence of cognitive decline.

\begin{tabular}{|c|c|c|c|c|c|c|c|c|c|}
\hline \multicolumn{5}{|c|}{ Predicting death in 2002} & \multicolumn{5}{|c|}{ Predicting death in 2004} \\
\hline & $B$ & SE & Wald & $\operatorname{Exp}(B) 95 \% \mathrm{CI}$ & & $B$ & SE & Wald & $\operatorname{Exp}(B) 95 \% \mathrm{CI}$ \\
\hline $2000 \mathrm{CVB}$ & .54 & .11 & $26.83^{\psi}$ & $1.40-2.11$ & $2002 \mathrm{CVB}$ & .43 & .12 & $11.97 \%$ & $1.21-1.97$ \\
\hline 2000 CESD & .08 & .04 & $3.90^{*}$ & $1.00-1.18$ & 2002 CESD & .17 & .05 & $13.71^{\psi}$ & $1.09-1.31$ \\
\hline 1998-2000 TICS >1SD & .40 & .23 & $2.92^{\S}$ & $.94-2.33$ & 2000-2002 TICS >1SD & .76 & .27 & $8.11^{+}$ & $1.27-3.61$ \\
\hline Constant & -2.49 & .18 & $183.52^{\psi}$ & & Constant & -2.68 & .22 & $146.83^{\psi}$ & \\
\hline \multicolumn{5}{|c|}{ Predicting death in 2006} & \multicolumn{5}{|c|}{ Predicting death in 2008} \\
\hline & $B$ & SE & Wald & $\operatorname{Exp}(B) 95 \% \mathrm{CI}$ & & $B$ & SE & Wald & $\operatorname{Exp}(B) 95 \% \mathrm{CI}$ \\
\hline 2004 CVB & .22 & .14 & 2.61 & $.95-1.64$ & 2006 CVB & .44 & .17 & $6.93^{+}$ & $1.12-2.16$ \\
\hline 2004 CESD & .03 & .06 & 0.29 & $.92-1.15$ & 2006 CESD & .07 & .07 & 1.09 & $0.94-1.22$ \\
\hline 2002-2004 TICS > 1SD & .39 & .34 & 1.36 & $.77-2.85$ & 2004-2006 TICS > 1SD & 1.12 & .43 & $6.86^{+}$ & $1.33-7.06$ \\
\hline Constant & -1.79 & .22 & 64.51 & & Constant & -2.15 & .30 & $50.13^{\psi}$ & \\
\hline
\end{tabular}

Note. CVB: cerebrovascular burden. CESD: Center for Epidemiological Studies Depression Scale. TICS: Telephone Interview for Cognitive Status. TICS Drop reflects incidence of decline on TICS score greater than 6 points between waves as indicated.

$\S P=.088,{ }^{*} P<.05 .{ }^{+} P<.01,{ }^{\psi} P<.001, \mathrm{df}=1$ for all comparisons.

aging theory, to greater longevity in this sample of older women. In the present study, Rowe and Kahn's formulation of successful aging is expanded from quality of life to length of life. Heightened depression and rapid loss of cognition were significantly related to timing of death, and conversely robust functioning was related to longevity. These findings underscore the significance of behavioral factors to the discussion of longevity. Behavioral factors such as mood and gross cognitive functioning are basic characteristics of the individual patient, and these findings highlight the importance of subjective reports or clinician perceptions of decline in these areas, especially when working with older patients. The finding that CVB predicts longevity broadly supports the large medical and epidemiological literature citing conditions such as heart disease, hypertension, and diabetes as risk factors for death (discussed in [11]). Our finding that depressive symptoms predict longevity corroborate significant past work on this subject [19-22].

These findings are also consistent with the concept of terminal cognitive drop [24]. While some studies support the terminal cognitive decline effect over longer periods of time
$[24,28,29]$, these findings support other research $[19,20]$ that terminal cognitive drop theory can be applied over brief periods of time. Additionally, most research supporting terminal cognitive decline relates mortality to impaired performance on cognitive measures, suggesting decline from an idiographic baseline and consequently discounting the time period over which decline occurs. By contrast, the present study contributes to the existing literature by testing this theory among older old women using longitudinal data.

The primary limitation of the present study is that cognitive and mood data are not available for many respondents approaching death. This data is absent largely for reasons of incapacity. Consequently, it is likely that the relationship between depressive symptoms, cognitive decline, and longevity is underrepresented by the present research. Another limitation is the use of categorical self-reported CVB data. However, use of such data is common in populationbased samples and reasonable concordance values between self-reports of disease and medical chart reviews have been reported [44, 45]. Future research should build on the present finding by identifying relationships between 
markers of cerebrovascular health, depression, and cognitive decline.

\section{Acknowledgments}

The development of this research was generously supported by the Blue Cross Blue Shield of Michigan Foundation and by the T32 grant-supported NIA/NIH Pre-Doctoral Training Program in Aging and Urban Health at the Institute of Gerontology. 2 T-32 AG00275-06.

\section{References}

[1] J. W. Rowe and R. L. Kahn, "Successful aging," The Gerontologist, vol. 37, no. 4, pp. 433-440, 1997.

[2] N. R. Cook, M. S. Albert, L. F. Berkman, D. Blazer, J. O. Taylor, and C. H. Hennekens, "Interrelationships of peak expiratory flow rate with physical and cognitive function in the elderly: MacArthur Foundation Studies of Aging," Journals of Gerontology—Series A, vol. 50, no. 6, pp. M317-M323, 1995.

[3] J. Chodosh, D. M. Kado, T. E. Seeman, and A. S. Karlamangla, "Depressive symptoms as a predictor of cognitive decline: MacArthur Studies of Successful Aging," The American Journal of Geriatric Psychiatry, vol. 15, no. 5, pp. 406-415, 2007.

[4] T. L. Gruenewald, A. S. Karlamangla, G. A. Greendale, B. H. Singer, and T. E. Seeman, "Feelings of usefulness to others, disability and mortality in older adults: the MacArthur Study of Successful Aging," Journals of Gerontology - Series B, vol. 62, no. 1, pp. P28-P37, 2007.

[5] W. J. Strawbridge, M. I. Wallhagen, and R. D. Cohen, "Successful aging and well-being: self-rated compared with Rowe and Kahn," The Gerontologist, vol. 42, no. 6, pp. 727-733, 2002.

[6] M. B. Holstein and M. Minkler, "Self, society and the 'New Gerontology”, The Gerontologist, vol. 43, no. 6, pp. 787-796, 2003.

[7] S. Arber and H. Cooper, "Gender differences in health in later life: the new paradox?" Social Science and Medicine, vol. 48, no. 1, pp. 61-76, 1999.

[8] A. L. Rosso, C. B. Eaton, R. Wallace et al., "Combined impact of geriatric syndromes and cardiometabolic diseases on measures of functional impairment," Journals of GerontologySeries A, vol. 66, no. 3, pp. 349-354, 2011.

[9] M. L. Bruce, T. E. Seeman, S. S. Merrill, and D. G. Blazer, "The impact of depressive symptomatology on physical disability: MacArthur Studies of Successful Aging," The American Journal of Public Health, vol. 84, no. 11, pp. 1796-1799, 1994.

[10] M. Hirvensalo, T. Rantanen, and E. Heikkinen, "Mobility difficulties and physical activity as predictors of mortality and loss of independence in the community-living older population," Journal of the American Geriatrics Society, vol. 48, no. 5, pp. 493-498, 2000.

[11] L. Flicker, "Cardiovascular risk factors, cerebrovascular disease burden and healthy brain aging," Clinics in Geriatric Medicine, vol. 26, no. 1, pp. 17-27, 2010.

[12] N. Raz, K. M. Rodrigue, and J. D. Acker, "Hypertension and the brain: vulnerability of the prefrontal regions and executive functions," Behavioral Neuroscience, vol. 117, no. 6, pp. 1169 1180, 2003.

[13] J. R. Sneed, D. Rindskopf, D. C. Steffens, K. R. Krishnan, and S. P. Roose, "The vascular depression subtype: evidence of internal validity," Biological Psychiatry, vol. 64, no. 6, pp. 491-497, 2008.
[14] C. E. Coffey, G. S. Figiel, W. T. Djang, and R. D. Weiner, "Subcortical hyperintensity on magnetic resonance imaging: a comparison of normal and depressed elderly subjects," The American Journal of Psychiatry, vol. 147, no. 2, pp. 187-189, 1990.

[15] A. H. Glassman and P. A. Shapiro, "Depression and the course of coronary artery disease," The American Journal of Psychiatry, vol. 155, no. 1, pp. 4-11, 1998.

[16] M. J. Bos, T. Lindén, P. J. Koudstaal et al., "Depressive symptoms and risk of stroke: the Rotterdam Study," Journal of Neurology, Neurosurgery and Psychiatry, vol. 79, no. 9, pp. 9971001, 2008.

[17] S. L. Larson, P. L. Owens, D. Ford, and W. Eaton, "Depressive disorder, dysthymia and risk of stroke: thirteen-year followup from the Baltimore Epidemiologic Catchment Area Study," Stroke, vol. 32, no. 9, pp. 1979-1983, 2001.

[18] B. W. Penninx, J. M. Guralnik, M. Pahor et al., "Chronically depressed mood and cancer risk in older persons," Journal of the National Cancer Institute, vol. 90, no. 24, pp. 1888-1893, 1998.

[19] C. L. Arfken, P. A. Lichtenberg, and M. E. Tancer, "Cognitive impairment and depression predict mortality in medically ill older adults," Journals of Gerontology-Series A, vol. 54, no. 3, pp. M152-M156, 1999.

[20] K. M. Mehta, K. Yaffe, K. M. Langa, L. Sands, M. A. Whooley, and K. E. Covinsky, "Additive effects of cognitive function and depressive symptoms on mortality in elderly communityliving adults," Journals of Gerontology-Series A, vol. 58, no. 5, pp. 461-467, 2003.

[21] K. E. Covinsky, E. Kahana, M. H. Chin, R. M. Palmer, R. H. Fortinsky, and C. S. Landefeld, "Depressive symptoms and 3year mortality in older hospitalized medical patients," Annals of Internal Medicine, vol. 130, no. 7, pp. 563-569, 1999.

[22] B. W. Penninx, S. W. Geerlings, D. J. Deeg, J. T. van Eijk, W. van Tilburg, and A. T. Beekman, "Minor and major depression and the risk of death in older persons," Archives of General Psychiatry, vol. 56, no. 10, pp. 889-895, 1999.

[23] P. Missotten, G. Squelard, M. Ylieff et al., "Quality of life in older Belgian people: comparison between people with dementia, mild cognitive impairment and controls," International Journal of Geriatric Psychiatry, vol. 23, no. 11, pp. 11031109, 2008.

[24] K. F. Riegel and R. M. Riegel, "Development, drop and death," Developmental Psychology, vol. 6, no. 2, pp. 306-319, 1972.

[25] E. Palmore and W. Cleveland, "Aging, terminal decline and terminal drop," Journals of Gerontology, vol. 31, no. 1, pp. 7681, 1976.

[26] H. B. Bosworth and I. C. Siegler, "Terminal change in cognitive function: an updated review of longitudinal studies," Experimental Aging Research, vol. 28, no. 3, pp. 299-315, 2002.

[27] M. F. Folstein, S. E. Folstein, and P. R. McHugh, "'Mini-mental state': a practical method for grading the cognitive state of patients for the clinician," Journal of Psychiatric Research, vol. 12, no. 3, pp. 189-198, 1975.

[28] K. Yaffe, K. Lindquist, E. Vittinghoff et al., "The effect of maintaining cognition on risk of sisability and death," Journal of the American Geriatrics Society, vol. 58, no. 5, pp. 889-894, 2010.

[29] R. S. Wilson, T. L. Beck, J. L. Bienias, and D. A. Bennett, “Terminal cognitive decline: accelerated loss of cognition in the last years of life," Psychosomatic Medicine, vol. 69, no. 2, pp. 131137, 2007.

[30] K. M. Langa, E. B. Larson, J. H. Karlawish et al., "Trends in the prevalence and mortality of cognitive impairment in the 
United States: is there evidence of a compression of cognitive morbidity?" Alzheimer's and Dementia, vol. 4, no. 2, pp. 134$144,2008$.

[31] B. T. Mast, B. Yochim, S. E. MacNeill, and P. A. Lichtenberg, "Risk factors for geriatric depression: the importance of executive functioning within the vascular depression hypothesis," Journals of Gerontology — Series A, vol. 59, no. 12, pp. 12901294, 2004.

[32] A. Bielak, D. Gerstorf, K. M. Kiely, K. J. Anstey, and M. Luszcz, "Depressive symptoms predict decline in perceptual speed in older adulthood," Psychology and Aging. In press.

[33] S. G. Heeringa and J. Conner, "Technical description of the Health and Retirement Study sample design," HRS/AHEAD Documentation Report DR-002, University of Michigan, Ann Arbor, Mich, USA, 1995.

[34] L. Radloff, "The CES-D Scale: a self-report depression scale for research in the general population," Applied Psychological Measurement, vol. 1, no. 3, pp. 385-401, 1977.

[35] D. E. Steffick, "Documentation of affective functioning measures in the Health and Retirement Study," HRS Documentation Report DR-005, Survey Research Center at the Institute for Social Research, Ann Arbor, Mich, USA, 2000.

[36] R. Wallace, A. R. Herzog, M. B. Ofstedal et al., "Documentation of affective functioning measures in the Health and Retirement Study," Tech. Rep., Survey Research Center, University of Michigan, Ann Arbor, Mich, USA, 2000.

[37] A. T. F. Beekman, D. J. H. Deeg, J. Van Limbeek, A. W. Braam, M. Z. De Vries, and W. Van Tilburg, "Criterion validity of the Center for Epidemiologic Studies Depression scale (CES-D): results from a community-based sample of older subjects in the Netherlands," Psychological Medicine, vol. 27, no. 1, pp. 231-235, 1997.

[38] J. Brandt, M. Spencer, and M. Folstein, "The telephone interview for cognitive status," Neuropsychiatry, Neuropsychology and Behavioral Neurology, vol. 1, no. 2, pp. 111-117, 1988.

[39] A. R. Herzog and R. B. Wallace, "Measures of cognitive functioning in the AHEAD study," Journals of Gerontology-Series $B$, vol. 52, special issue, pp. 37-48, 1997.

[40] K. A. Welsh, J. C. S. Breitner, and K. M. Magruder-Habib, "Detection of dementia in the elderly using telephone screening of cognitive status," Neuropsychiatry, Neuropsychology and Behavioral Neurology, vol. 6, no. 2, pp. 103-110, 1993.

[41] D. W. Desmond, T. K. Tatemichi, and L. Hanzawa, "The telephone interview for cognitive status (TICS): reliability and validity in a stroke sample," International Journal of Geriatric Psychiatry, vol. 9, no. 10, pp. 803-807, 1994.

[42] T. Järvenpää, J. O. Rinne, I. Räihä et al., "Characteristics of two telephone screens for cognitive impairment," Dementia and Geriatric Cognitive Disorders, vol. 13, no. 3, pp. 149-155, 2002.

[43] C. L. Dahle, B. S. Jacobs, and N. Raz, "Aging, vascular risk and cognition: blood glucose, pulse pressure and cognitive performance in healthy adults," Psychology and Aging, vol. 24, no. 1, pp. 154-162, 2009.

[44] T. L. Bush, S. R. Miller, A. L. Golden, and W. E. Hale, "Selfreport and medical record report agreement of selected medical conditions in the elderly," The American Journal of Public Health, vol. 79, no. 11, pp. 1554-1556, 1989.

[45] B. M. Psaty, L. H. Kuller, D. Bild et al., "Methods of assessing prevalent cardiovascular disease in the cardiovascular health study," Annals of Epidemiology, vol. 5, no. 4, pp. 255-335, 1995. 


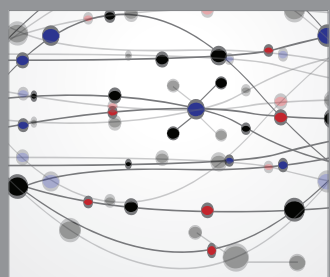

The Scientific World Journal
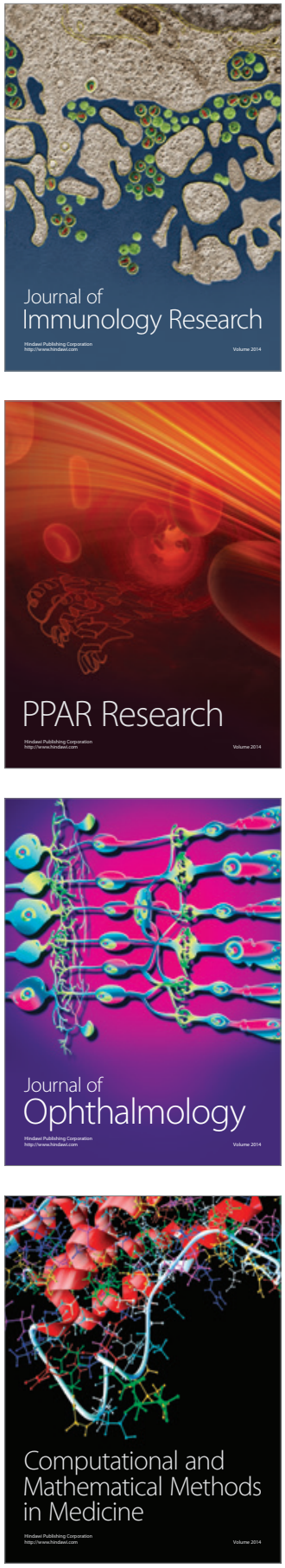

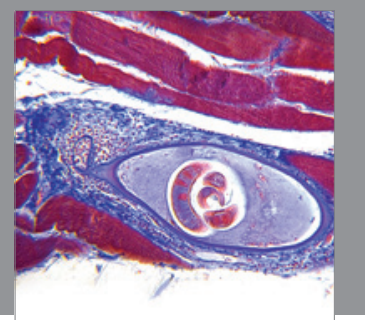

Gastroenterology

Research and Practice
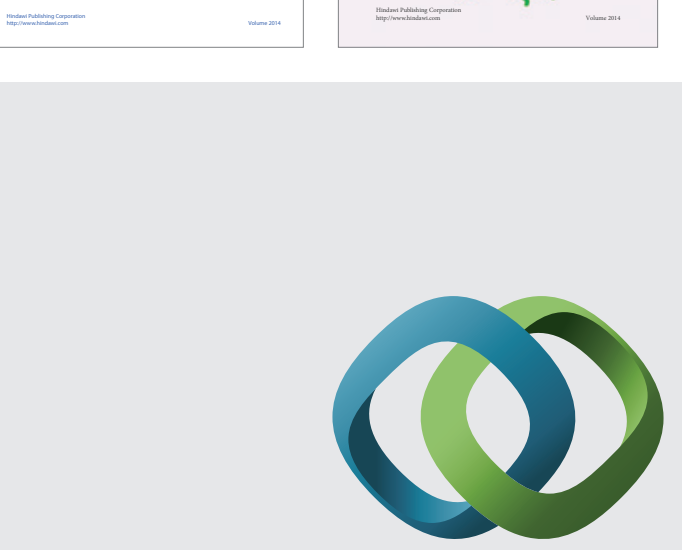

\section{Hindawi}

Submit your manuscripts at

http://www.hindawi.com
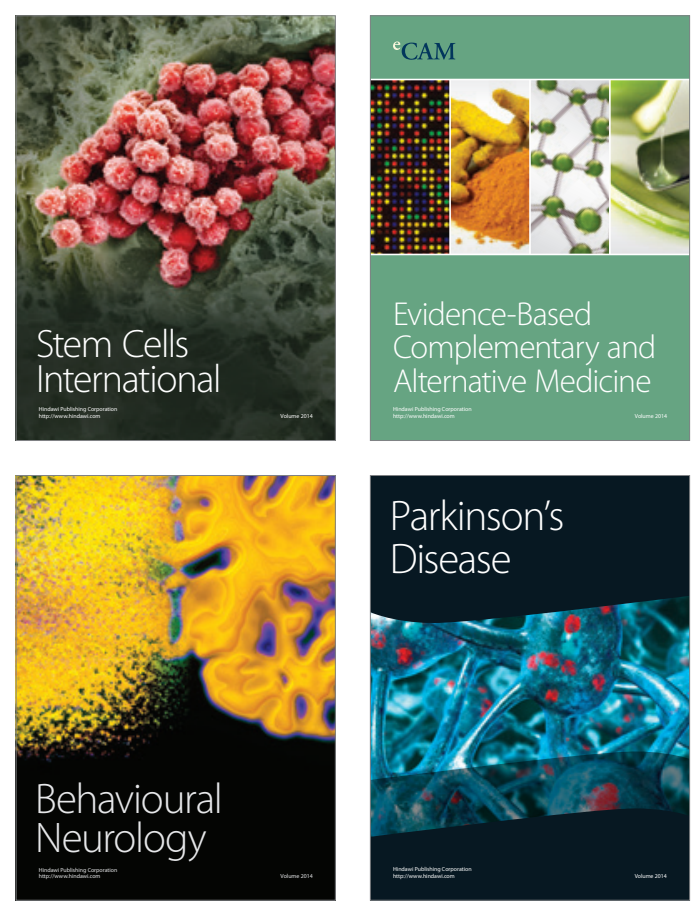

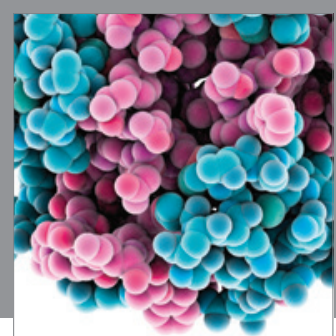

Journal of
Diabetes Research

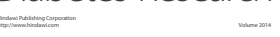

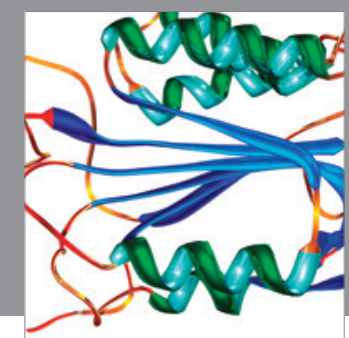

Disease Markers
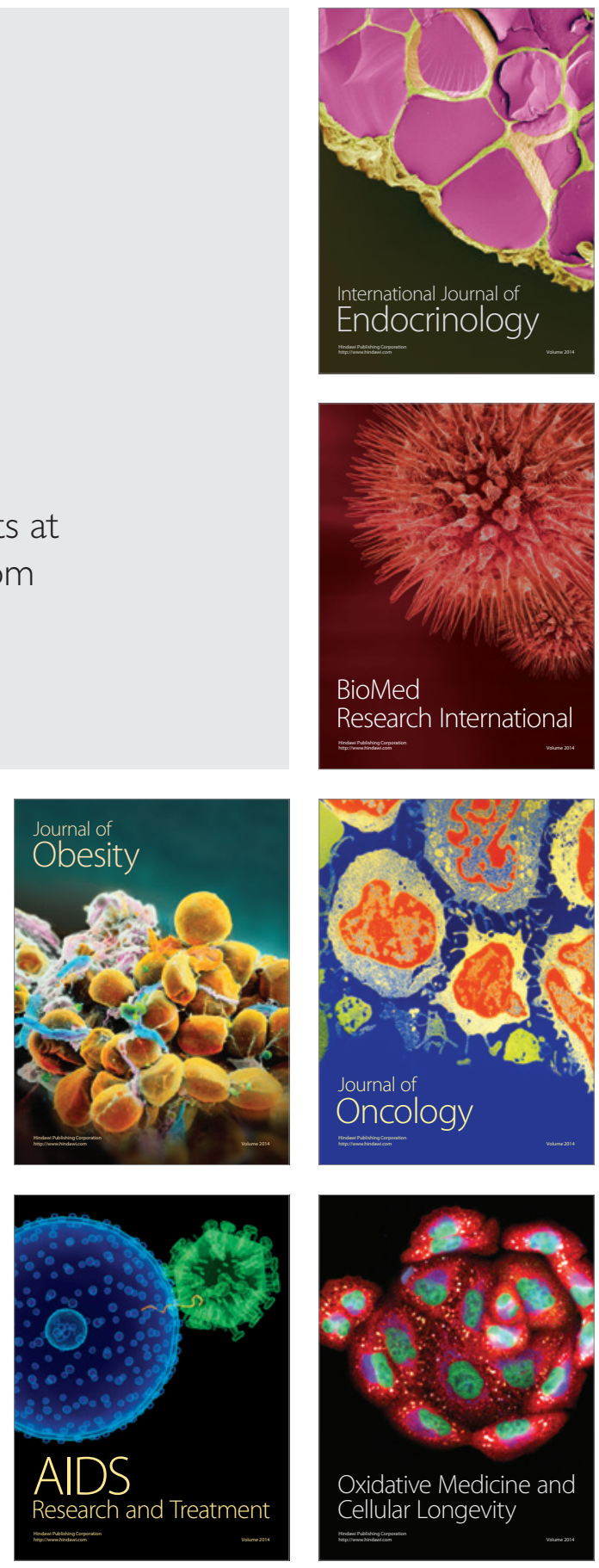